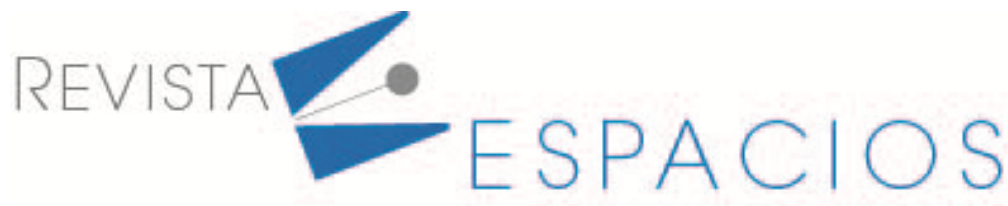

\title{
Adulto mayor en Cartagena de Indias -Colombia- necesidades, gustos y preferencias por el calzado
}

\section{Senior citizen in Cartagena de Indias -Colombia-: needs, interests and preferences for footwear}

\author{
LONDOÑO ALDANA, Emperatriz ${ }^{1}$ \\ NAVAS RÍOS, María Eugenia ${ }^{2}$ \\ PÉREZ RÍOS, Wilson E. ${ }^{3}$
}

\begin{abstract}
Resumen
La presente investigación busca conocer las necesidades, gustos y preferencias de las mujeres adultas mayores en cuanto al calzado. La información inicial fue obtenida de 12 grupos focales y 42 entrevistas. Luego se aplicó un formulario estructurado con objetivo no encubierto a 144 mujeres de 60 años y más. Se hizo un análisis uní y bivariado con aplicación de prueba $\mathrm{F}$ de Snedecor para establecer diferencias significativas. Igualmente, se ejecutó una red neuronal artificial de clasificación.

Palabras claves: adulto mayor, calzado, juanetes, callos, dedos de martillo
\end{abstract}

\begin{abstract}
This research seeks to know the needs, interests and preferences of older women in terms of footwear. The initial information was obtained from 12 focus groups and 42 interviews. Then a structured, nonblinded objective form was applied to 144 women aged 60 years and older. A uní and bivariate analysis was carried out with application of Snedecor F-test to establish significant differences. Likewise, an artificial neural network of classification was executed.
\end{abstract}

Keywords: seniors citizens, footwear, bunions, corns, hammertoes

\section{Introducción}

Los pies tienen la capacidad de soportar el peso de las personas a lo largo de sus vidas y están en constante actividad durante el día, bien sea que se esté de pie, en movimiento o incluso sentado, con el consecuente esfuerzo que en condiciones normales solamente puede ser contrarrestado con un acorde con su respectiva morfología. Sin embargo, a ellos no siempre se les da la importancia y cuidados necesarios en función de las actividades diarias; igualmente no se les prepara para las situaciones que hay que enfrentar y afrontar en la adultez, toda vez que tanto la estética como la moda priman sobre la comodidad y seguridad del calzado principalmente en las mujeres-; ello bajo la premisa de que este es un accesorio indispensable en la indumentaria requerida en la presentación personal dejando de lado su funcionalidad referida a la protección.

\footnotetext{
${ }^{1}$ Docente investigador. Universidad de Cartagena. elondonoa@unicartagena.edu.co

${ }^{2}$ Docente investigador. Universidad de Cartagena. mnavasr@unicartagena.edu.co

${ }^{3}$ Asistente de investigación Universidad de Cartagena. wperezr@unicartagena.edu.co
} 
De otra parte, las personas se han acostumbrado a elegir su calzado en función de la comodidad, sin considerar su seguridad y el mayor riesgo de caídas. Muchos adultos mayores creen les va bien comprando zapatos ligeros y suaves que son fáciles de usar ponerse y quitarse.

La función del pie durante un paso, se puede dividir en tres fases básicas: primero, en el contacto inicial del talón con el suelo, el pie se encarga de frenar el descenso de la masa corporal para, a continuación, con todo el pie apoyado, servir de base para la progresión del cuerpo y, finalmente, permitir la impulsión hacia delante perdiendo el contacto con el suelo por los dedos. La marcha de los mayores se encuentra sometida a la influencia de los cambios propios de la edad y a los efectos de las diversas patologías de aparición frecuente en estos grupos etarios (Marco, 2003). Así, las consecuencias de un calzado inadecuado son: callos, durezas, juanetes, dedos en garra, entre otras patologías. Adicional a ello, la piel es la primera barrera que tiene el cuerpo y es la primera que sufre; un callo, por ejemplo, no es más que una defensa de la piel contra algo que le roza. En el adulto mayor, dichas consecuencias, se agudizan llegando a serias patologías, por tanto, se hace necesario contar con una oferta de calzado acorde con la morfología y particularidades de los pies, lo cual apunta tanto a la comodidad y calidad de vida del usuario como a la prevención de enfermedades que son del tenor de la Podología y otras especiales.

Si bien existen fabricantes de calzado que miran hacia el mercado de la tercera edad, se focalizan principalmente en diseños para la satisfacción solo de algunas necesidades, pero exentos de estética y elegancia; han dejado por fuera los gustos y preferencias de los usuarios olvidándose que cualquiera que sea la edad las personas, ellas desean sentirse no solo cómodas sino bien presentadas.

Acorde con lo anterior, en el trabajo se analizan las necesidades, gustos y preferencias de mujeres con 60 años y más en materia de calzado.

\section{Referentes teóricos}

La población envejece y de acuerdo con la Organización Mundial de la Salud (2017), el incremento de adultos mayores hacia 2050 fluctuará entre el 12\% y 22\%. En Colombia los resultados del censo 2018 realizado por el Departamento Nacional de Estadística DANE, indican que el 9,1\% corresponde a mayores de 65 años, con una proyección a 2050 del 23\%, mostrando un fuerte adelgazamiento de la correspondiente pirámide poblacional.

El envejecimiento de las personas, implica una serie de cambios fisiológicos, emocionales y mentales así, el adulto mayor experimenta importantes cambios en relación con su constitución corporal que determinan que su fisiología sea muy distinta a la de los sujetos en edad media de la vida (Salech, et., alia., 2012). Dentro de los cambios fisiológicos anotados, se encuentran la disminución de la masa muscular y aumento de la infiltración grasa asociada a una reducción progresiva de la fuerza, decoloración del cabello, piel arrugada, espalda encorvada, disminución de estatura, huesos, músculos y articulaciones, afectan la postura y la marcha y llevan a debilidad y lentitud en los movimientos.

Según Romero, et., alia., (2010), en la Podología, la mayor parte de los usuarios son personas de edad avanzada quienes por falta de movilidad o medios, son incapaces de llevar a cabo el correcto cuidado de sus pies con la consecuente afectación en su bienestar. En estas personas se agudizan los factores podológicos dados los problemas fisiológicos que son propios de la edad, como deshidratación, piel seca, disminución de las fuerzas activas musculares, y la forma del pie que tiende a alterarse con la edad, siendo este último un problema muy común en la mayoría de ellas lo que les genera dificultades al caminar, hinchazón, inestabilidad, desgaste del colchón natural del pie, limitación a la movilidad, etc.

Los cambios en la morfología del pie, de acuerdo con Anzuátegui, et., (2016), ocurren con el envejecimiento y tienden a ser diferenciales entre hombres y mujeres, en el marco de las tipologías propias de cada etnia. Los 
arcos de los pies se vuelven menos pronunciados, lo que contribuye a una pérdida ligera de estatura; además, el riesgo de lesión se incrementa debido a que los cambios en la marcha, inestabilidad y pérdida del equilibrio pueden conducir a caídas.

Los pies de los mayores, anotan Droulers y Goldcher (2009), acumulan sufrimiento que es esencialmente de origen vascular, osteoarticular, cutáneo, tendinomuscular o neurogénico lo que hace necesario el uso de calzado que se adapte a sus necesidades o que se fabrique a medida, ortesis plantares u ortoplastias (apoyo u otro dispositivo externo aplicado al cuerpo para modificar los aspectos funcionales o estructurales del sistema neuromusculoesquelético).

El Instituto Valenciano del Pie, destaca los siguientes aspectos propios de la edad que influyen sobre la marcha de las personas mayores:

- Disminución de las fuerzas activas musculares responsables del movimiento. Esto genera que la impulsión sea menos vigorosa.

- Los ligamentos y el complejo capsular se vuelven más rígidos y el cartílago articular pierde sus propiedades mecánicas. La amortiguación de impactos, durante la marcha, es menor.

- Reducción de la capacidad de movimiento con la edad, de efecto mucho mayor en las mujeres que en los hombres.

- Pérdida de agudeza visual, que genera problemas de equilibrio. La visión constituye un sistema sensorial de importancia extrema para mantener el equilibrio durante la marcha.

- Alteraciones frecuentes entre los mayores como la artritis y la obesidad, contribuyen a la aparición de trastornos de la marcha.

- El uso prolongado del tacón alto por las mujeres puede provocar un acortamiento del tendón de Aquiles por lo que la dorsiflexión es menor en mujeres y la flexión plantar mayor que en los hombres. Esto impide que muchas mujeres mayores puedan utilizar calzado bajo.

- La posición del cuerpo, ligeramente flexionada hacia delante, provoca el desplazamiento del centro de gravedad en sentido anterior, y dificulta el mantenimiento de la posición erecta.

Chaiwanichsiri, et., al., (2008), señalan que los fabricantes de calzado aún confían en hormas diseñadas para población adulta más joven, mientras que la morfología de los pies de los mayores es claramente diferente de la de los pies jóvenes. El tipo de calzado usado por las personas mayores es uno de los factores de riesgo para caída y no todos los zapatos tienen las mismas características en términos de prevención de caídas (Carbillet, 2012).

Para el acople del pie dentro entro del calzado se recurre a la variedad de complementos tales como: plantillas ortopédicas elaboradas con un material flexible o rígido según la necesidad bien sean prefabricados o moldeados, ortesis plantares elaboradas en material más rígido y por ende más correctivo, adaptaciones en suelas u ortesis, almohadillas retrometatarsiana, bisel pronador, bisel supinador, bisel interno y externo, almohadilla para elevar el talón y zapatos adaptados elaborados en suela flexible, talón acampanado, moldeado y confeccionado a la medida (Harvey, 2001).

En concordancia con lo anterior la búsqueda de zapatos cómodos y "saludables" es vital para las personas de la tercera edad. Dicho calzado debe satisfacer las necesidades como lo son la protección frente al ambiente, seguridad, comodidad, flexibilidad y resistencia a los golpes. Sin embargo, Harvey (2001) comenta que muchas mujeres consideran que "a su edad no vale la pena pagar por buenos zapatos". Destaca las personas mayores que viven en la línea de pobreza, ya no invierten en zapatos cómodos que apunten a una mejor calidad de vida y tienden a calzar los zapatos usados por mucho tiempo, chancletas o pantuflas que para su edad no ofrecen un buen soporte o estabilidad. 
Especialistas en la confección de zapatos para adultos mayores conceptúan que la elección del calzado, para ellos, tiene un punto de dificultad extra que se añade a la correcta elección de calzado para pies delicados. De acuerdo con White y Mulley (1989), los cambios fisiológicos afectan la forma de andar y generan limitantes para calzarse y descalzarse, por lo que recurren a zapatos holgados o zapatillas, especialmente dentro y alrededor de la casa; agregan que aunque no se tenga ningún problema específico, se hace necesario un zapato especial para pies delicados que se adapte a su forma de caminar y por ende a evitar caídas, lesiones en los dedos y en la planta del pies como consecuencia de una presión inadecuada.

Jellema, et., alia., (2019), señalan que la fabricación de calzado estándar presenta limitaciones por cuanto las dimensiones entre los dos pies puede ser sensiblemente diferente; en consecuencia, el ajuste adecuado del calzado es fundamental toda vez que tiene un impacto positivo en la calidad de vida; así los zapatos que son demasiado estrechos o demasiado pequeños pueden causar dolor y los demasiados flojos facilitan resbalones y caídas. Fabricantes especializados han mejorado el ajuste y comodidad del calzado mediante la utilización de dispositivos de medición como el Brannock que es la herramienta de medición de pies estándar para la industria del calzado en todo el mundo,. o el Shimano.

El tipo de plantilla en el calzado juega igualmente un papel importante; Losa, et., al., (2012), indican que estas mejoran la función somato-sensorial, debido a una mejor alineación de las articulaciones de los pies, mejorando potencialmente las contribuciones musculares a la estabilidad y la distribución de la presión plantar.

Un calzado óptimo para adultos mayores debe contar con un ajuste anatómico adecuado, una puntera bien ajustada, un talón limitado altura, un talón lo suficientemente ancho, una plantilla y entresuela firmes, una suela exterior con banda de rodadura y ancho suficientes, un talón biselado, una punta de zapato biselada, un firme. Talonera con ajuste ceñido y mecanismo de cierre fácil y efectivo (Jellema, et., alia.,2019).

En el cuadro 1., se resume los cambios que los mayores presentan en sus pies y que deben tenerse en cuenta tanto en la fabricación como en la adquisición de calzado para personas de la tercera edad.

Tabla 1

Cambios morfológicos del pie en adultos mayores

\begin{tabular}{|c|c|c|}
\hline Cambios & $\begin{array}{l}\text { Características / } \\
\text { Qué se debe evitar }\end{array}$ & Tipo de calzado \\
\hline $\begin{array}{c}\text { Inestabilidad de la marcha y } \\
\text { reducción de la capacidad de } \\
\text { movimiento } \\
\text { Alto riesgo a deslizarse } \\
\text { lateralmente } \\
\text { Pérdida de agudeza visual } \\
\text { Disminución de la capacidad } \\
\text { para inclinarse hacia adelante } \\
\text { o hacia atrás mientras se está } \\
\text { de pie. }\end{array}$ & $\begin{array}{l}\text { Posibles resbalones y caídas } \\
\text { Daños en el talón } \\
\text { Problema de equilibrio durante } \\
\text { la marcha o estático. }\end{array}$ & $\begin{array}{l}\text { Calzado que se adapte perfectamente a los } \\
\text { movimientos del pie } \\
\text { Calzado con suelas que aporten un agarre máximo a la } \\
\text { superficie } \\
\text { Zapato con collar alto (altura del talón hasta el tobillo). }\end{array}$ \\
\hline
\end{tabular}




\begin{tabular}{|c|c|c|}
\hline Cambios & $\begin{array}{l}\text { Características / } \\
\text { Qué se debe evitar }\end{array}$ & Tipo de calzado \\
\hline & & $\begin{array}{l}\text { Plantillas y calzado a medida. La adaptación del pie al } \\
\text { calzado contribuye a una mejor salud física y mental. }\end{array}$ \\
\hline Dolencias propias de la edad & $\begin{array}{l}\text { Algunas patologías como la } \\
\text { artrosis pueden ser muy } \\
\text { dolorosas al caminar. }\end{array}$ & $\begin{array}{l}\text { Calzado que se adapte a las propias dolencias de la } \\
\text { persona. } \\
\text { Calzado que proporcione distribución de las presiones } \\
\text { en la planta }\end{array}$ \\
\hline $\begin{array}{l}\text { Disminución de la respuesta } \\
\text { muscular } \\
\text { Rigidez en los ligamentos y } \\
\text { articulaciones }\end{array}$ & $\begin{array}{l}\text { Menor amortiguación de } \\
\text { impactos }\end{array}$ & $\begin{array}{l}\text { Calzado que amortigüe correctamente los impactos } \\
\text { contra el suelo para evitar daños en el talón. }\end{array}$ \\
\hline $\begin{array}{l}\text { Sequedad en piel y uñas } \\
\text { y disminución de la agudeza } \\
\text { sensitiva }\end{array}$ & $\begin{array}{c}\text { Aumento de la fragilidad de las } \\
\text { uñas. } \\
\text { Peladuras e irritación con riesgo } \\
\text { de infección } \\
\text { Provocación de heridas }\end{array}$ & $\begin{array}{l}\text { Calzado sin costuras interiores } \\
\text { Si hay costuras que los remates sean totalmente planos }\end{array}$ \\
\hline $\begin{array}{l}\text { Ensanchamiento del pie } \\
\text { Presencia de juanetes } \\
\text { Alteraciones en los dedos } \\
\text { debido al uso del calzado } \\
\text { inadecuado, como el uso } \\
\text { prolongado de tacón por } \\
\text { parte de las mujeres } \\
\text { Dedos en garra o en martillo. }\end{array}$ & $\begin{array}{l}\text { Dolencias } \\
\text { Alteración de la circulación } \\
\text { Hinchazón }\end{array}$ & $\begin{array}{l}\text { Calzado flexible que se adapte perfectamente a la forma } \\
\text { del pie, para reducir al máximo las presiones y por tanto } \\
\text { los daños que puedan provocar un zapato incorrecto. }\end{array}$ \\
\hline
\end{tabular}

Fuente: Losa, et., al., (2012), Jellema, et., alia.,2019, White y Mulley (1989), Salech, et., alia., 2012. Marco (2003), Maki, et., al., (1999), Robbins, et., al. (1997) y Menz y Lord (1999).

\section{Metodología}

El presente trabajo corresponde a una investigación descriptiva de corte cuali-cuantitativo en el que utilizó el método transversal de encuestas y se llevó a cabo en tres etapas:

Etapa 1. Observación a mujeres adultas mayores en la calle (forma de caminar, tipo de calzado que usan) y en establecimientos que ofertan calzado (tipo de calzado escogido, expresiones y preguntas vendedores).

Etapa 2. Entrevistas a mujeres adultas mayores y realización de 12 focus group de ocho mujeres cada uno.

Etapa 3. Aplicación de un formulario estructurado con objetivo no encubierto a 144 mujeres con 60 años y más. Se optó por un muestreo por cuotas no probabilístico. De la observación, entrevistas y focus group, se derivaron preguntas categóricas y mixtas.

La estructura de la muestra tiene las siguientes características: ligeramente por encima del $50 \%$ se encuentran las mujeres con edades entre 60 y 70 años; le sigue un 30,56\% con edades entre 71 y 80 años y solo un 15,97\% con edades entre 80 y 90 años. En cuanto a la ocupación u oficio, el 4I,61\% corresponde a amas de casa, 20,14\% trabajadora independiente, $18,75 \%$ empleada y 12,50 pensionada. La distribución de los diferentes tipos de pie identificados corresponde a 33,33\% egipcio, 19,44\% germánico, $17,26 \%$, cuadrado $13,89 \%$, griego 10,42\% y celta 5,56\%. Figura 1. 
Figura 1

Distribución porcentual de mujeres según el tipo de pie
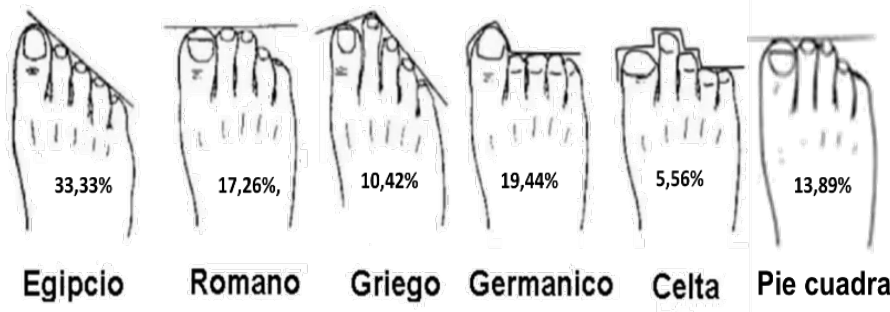

Griego Germanico

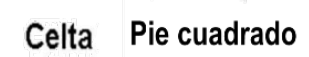

Se utilizó una escala de Likert con valoración de 5 puntos para medir grados de acuerdo/desacuerdo en las necesidades, gustos y preferencias por el calzado. El grado de confiabilidad obtenido con la prueba Alpha de Cronbach fue de $83 \%$. Se llevó a cabo un análisis uni variado y bivariado con aplicación de la prueba F de Snedecor y una red neuronal artificial. Para los efectos se aplicó el software Dyane 4 de Miguel Santesmases Mestre (2009).

\section{Resultados y discusión}

Al ser Cartagena de Indias una ciudad tropical costera en donde la temperatura media durante todo el año fluctúa en 28 y 35 grados, el tipo de calzado utilizado por las personas en su mayoría es abierto lo que favorece la comodidad del pie. Durante la observación en la fase exploratoria, se encontró que para las mujeres adultas mayores se presenta en el mercado una muy exigua oferta de calzado tanto abierto como cerrado acorde tanto con su edad como la morfología y patologías de sus pies.

Tabla 1

Características Afecciones del del pie

\begin{tabular}{ccccc}
\hline Código & Significado (respuestas múltiples) & Frecuencias & $\%$ s/ total frec. & $\%$ s/ muestra \\
\hline 1 & Juanetes & 27 & 13,17 & 18,75 \\
2 & Uñas encarnadas & 25 & 12,20 & 17,36 \\
3 & Callos & 37 & 18,05 & 25,69 \\
4 & Dedos en martillo & 14 & 6,83 & 9,72 \\
5 & Dedo en garra & 2 & 0,98 & 1,39 \\
6 & Dedo en mazo & 2 & 0,98 & 1,39 \\
7 & Piel delicada al roce con el zapato & 5 & 2,44 & 3,47 \\
8 & Grietas en los talones & 3 & 1,46 & 2,08 \\
9 & Dolor en los talones & 4 & 1,95 & 2,78 \\
10 & Pie plano & 4 & 1,95 & 2,78 \\
11 & Pie cavo & 2 & 0,98 & 1,39 \\
12 & Pie demasiado delgado & 3 & 1,46 & 2,08 \\
13 & Pie demasiado grueso & 7 & 3,41 & 4,86 \\
14 & Ninguna & 41 & 20,00 & 28,47 \\
15 & Otra & 29 & 14,15 & 20,14 \\
\hline \multicolumn{5}{r}{} \\
\hline
\end{tabular}

Fuente: Encuestas - base de datos Bernard K. (2020).

Adulto mayor en Cartagena: necesidades, gustos y preferencias por el calzado

Las principales características de los pies de las mujeres mayores son en su orden, callos (18,05\%), juanetes $(13,17 \%)$ y uñas encarnadas $(12,20 \%)$ como lo afirman el $25,69 \%, 18,75 \%$ y $17,36 \%$ respectivamente. Estos 
aspectos tienen su explicación principalmente por el tipo de calzado utilizado durante su juventud, el cual se caracterizaba una puntera aguda y un tacón alto muy delgado. (Tabla 1.).

\subsection{Necesidades}

Debido a los cambios morfológicos a causa de su edad, los adultos mayores desarrollan problemas en sus pies que les obliga a calzar zapatos con materiales, hormas y diseños que se acomoden a sus necesidades. De acuerdo con las valoraciones dadas (4,1 a 4,9 tabla 2), ellos piensan que el calzado debería estar acorde con las características del pie, coinciden que si es liviano evita el cansancio y brinda seguridad, además consideran que es difícil conseguir zapatos cómodos que no afecte sus juanetes y que si bien su horma amplia ayuda a quienes padecen dedos de martillo, garra o mazo esta es muy difícil de conseguir en la oferta actual de la ciudad. Estas percepciones analizadas en función de la profesión u oficio son similares en todos los apartados en el sentido en que las valoraciones, cualesquiera que sean estas, se encuentran por encima de cuatro.

Es importante destacar que en lo referente a la fascia plantar la valoración se encuentra en el rango de indiferencia $(3,6)$. Al analizar esta percepción en función de la ocupación oficio, se observa los empleados $(3,8)$, amas de casa $(3,7)$ y trabajadores independientes $(3,7)$ son quienes perciben la necesidad de calzado suave y que el forrado sea de almohadilla con el fin de evitar las dolencias en los talones y planta del pie producto de la fascia plantar en cambio los pensionados se encuentran en un nivel neutro o de indiferencia $(3,0)$; esto es explicable por cuanto no tienen la movilidad permanente de los demás.

Tabla 2

Necesidades por el tipo de calzado según la profesión u oficio

\begin{tabular}{|c|c|c|c|c|c|c|c|}
\hline \multirow[b]{2}{*}{$\begin{array}{l}\text { № } \\
\text { Var. }\end{array}$} & \multirow[b]{2}{*}{ Denominación } & \multirow[b]{2}{*}{$\begin{array}{c}\text { Total } \\
\text { muestra }\end{array}$} & \multicolumn{4}{|c|}{ Ocupación u oficio } & \multirow[b]{2}{*}{ F de Snedecor } \\
\hline & & & Ama casa & $\begin{array}{l}\text { Traba. } \\
\text { Indep. }\end{array}$ & Empl. & Pens. & \\
\hline 10 & Juanetes: calzado suave. & $\begin{array}{l}3,2986 \\
n=144\end{array}$ & $\begin{array}{c}3,4429 \\
n=70\end{array}$ & $\begin{array}{l}2,8621 \\
n=29\end{array}$ & $\begin{array}{l}3,3704 \\
n=27\end{array}$ & $\begin{array}{l}3,3333 \\
n=18\end{array}$ & $\begin{array}{c}F(3,140)=1,7637 \\
p=0,1569\end{array}$ \\
\hline 11 & Juanetes: sandalias & $\begin{array}{l}3,7778 \\
n=144\end{array}$ & $\begin{array}{c}3,8714 \\
n=70\end{array}$ & $\begin{array}{l}3,5517 \\
n=29\end{array}$ & $\begin{array}{l}3,7407 \\
n=27\end{array}$ & $\begin{array}{l}3,8333 \\
n=18\end{array}$ & $\begin{array}{c}F(3,140)=0,4893 \\
p=0,6902\end{array}$ \\
\hline 12 & Juanetes: no hay calzado cerrado cómodo & $\begin{array}{l}4,1319 \\
n=144\end{array}$ & $\begin{array}{l}4,2286 \\
n=70\end{array}$ & $\begin{array}{l}4,0345 \\
n=29\end{array}$ & $\begin{array}{l}4,0741 \\
n=27\end{array}$ & $\begin{array}{l}4,0000 \\
n=18\end{array}$ & $\begin{array}{c}F(3,140)=0,3859 \\
p=0,7633\end{array}$ \\
\hline 14 & Calzado acorde a las características del pie & $\begin{array}{l}4,9306 \\
n=144\end{array}$ & $\begin{array}{l}4,9143 \\
n=70\end{array}$ & $\begin{array}{l}4,8966 \\
n=29\end{array}$ & $\begin{array}{l}4,9630 \\
n=27\end{array}$ & $\begin{array}{l}5,0000 \\
n=18\end{array}$ & $\begin{array}{c}F(3,140)=0,3692 \\
p=0,7754\end{array}$ \\
\hline 15 & Fascia plantar: calzado suave y abullonado & $\begin{array}{l}3,6389 \\
n=144\end{array}$ & $\begin{array}{c}3,6714 \\
n=70\end{array}$ & $\begin{array}{l}3,6897 \\
n=29\end{array}$ & $\begin{array}{l}3,8889 \\
n=27\end{array}$ & $\begin{array}{l}3,0556 \\
n=18\end{array}$ & $\begin{array}{c}F(3,140)=2,8743 \\
p=0,0385\end{array}$ \\
\hline 16 & $\begin{array}{l}\text { Calzado liviano evita cansancio y permite } \\
\text { seguridad. }\end{array}$ & $\begin{array}{l}4,7917 \\
n=144\end{array}$ & $\begin{array}{l}4,7857 \\
n=70\end{array}$ & $\begin{array}{l}4,7586 \\
n=29\end{array}$ & $\begin{array}{l}4,7407 \\
n=27\end{array}$ & $\begin{array}{l}4,9444 \\
n=18\end{array}$ & $\begin{array}{c}F(3,140)=0,6946 \\
p=0,5568\end{array}$ \\
\hline 17 & Horma de calzado amplia & $\begin{array}{l}4,5764 \\
n=144\end{array}$ & $\begin{array}{l}4,6143 \\
n=70\end{array}$ & $\begin{array}{l}4,4483 \\
n=29\end{array}$ & $\begin{array}{l}4,7407 \\
n=27\end{array}$ & $\begin{array}{l}4,3889 \\
n=18\end{array}$ & $\begin{array}{c}F(3,140)=1,6999 \\
p=0,1698\end{array}$ \\
\hline 18 & Calzado con suela antideslizante. & $\begin{array}{l}4,8542 \\
n=144\end{array}$ & $\begin{array}{l}4,8714 \\
n=70\end{array}$ & $\begin{array}{l}4,8276 \\
n=29\end{array}$ & $\begin{array}{l}4,8889 \\
n=27\end{array}$ & $\begin{array}{l}4,7778 \\
n=18\end{array}$ & $\begin{array}{c}F(3,140)=0,3515 \\
p=0,7881\end{array}$ \\
\hline 19 & $\begin{array}{l}\text { Calzado para pies con dedos: martillo, } \\
\text { garra y mazo. }\end{array}$ & $\begin{array}{l}4,7986 \\
n=144\end{array}$ & $\begin{array}{l}4,7857 \\
n=70\end{array}$ & $\begin{array}{l}4,8621 \\
n=29\end{array}$ & $\begin{array}{l}4,7778 \\
n=27\end{array}$ & $\begin{array}{l}4,7778 \\
n=18\end{array}$ & $\begin{array}{c}F(3,140)=0,1469 \\
p=0,9315\end{array}$ \\
\hline
\end{tabular}

Fuente: Encuestas - base de datos Bernard K. (2020).

Adulto mayor en Cartagena: necesidades, gustos y preferencias por el calzado

Lo expuesto se confirma con la aplicación de la prueba F de Snedecor cuyo nivel de significancia se encuentra al nivel del 5\%, por tanto, se afirma que la necesidad de calzado suave y con almohadilla debido a padecimiento de fascia plantar depende de la ocupación u oficio que desempeña el adulto mayor. En los demás casos no se presentan diferencias significativas como la prueba $\mathrm{F}$ de Snedecor que en todos los casos el nivel de significancia está por encima del $5 \%$. 


\subsection{Clasificación de necesidades de calzado - red neuronal artificial}

Para una mejor clasificación de las necesidades por el tipo de calzado, en relación con las actividades que realizan los adultos mayores, se optó por una red neuronal artificial (RNA) utilizando el modelo de clasificación. Fueron tomadas como variables output las categorías de la ocupación u oficio y como las variables input las necesidades por el calzado acorde con las características y patologías del pie.

El modelo (RNA) perceptrón multicapa, cuenta con tres capas y trece variables en total: nueve de entrada y cuatro de salida; por ende, se fija en seis el número de variables intermedias u ocultas -semisuma del total de variables de input y output-. Figura 1.

Componentes del modelo:

- Numero de interacciones: 5003

- Tasa de aprendizaje inicial: 1,00

- Tasa de aprendizaje final: 0,70

- Momentos: Inicial: 0,00 - Final: 0,30

- Conjunto de entrenamiento: casos 1 a 144

- Conjunto de Evaluación: casos 1 a 144

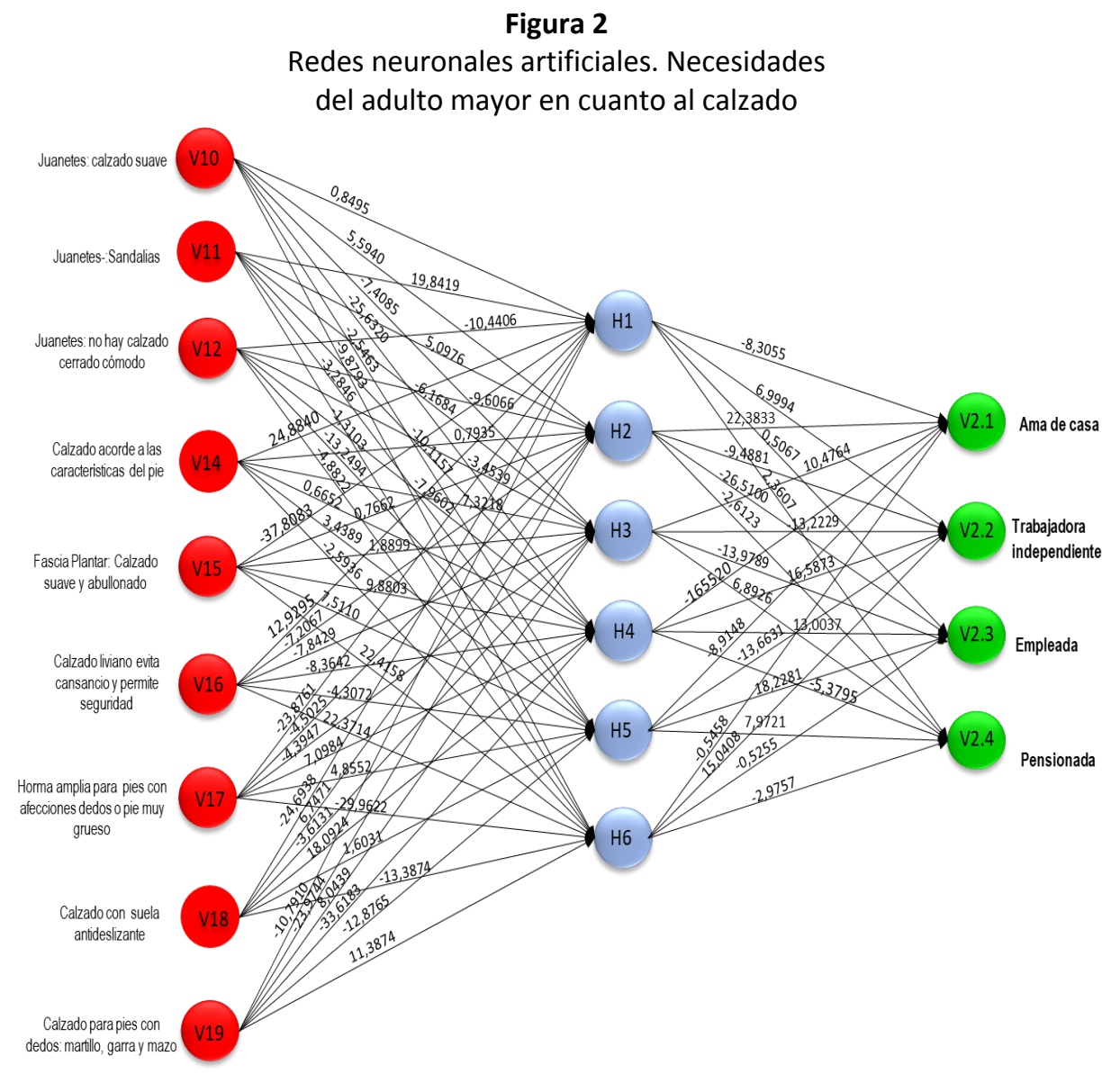

Fuente: Fuente: Encuestas - base de datos Bernard K. (2020).

Adulto mayor en Cartagena: necesidades, gustos y preferencias por el calzado

En la matriz de confusión obtenida en el análisis (tabla 3), las estimaciones de los valores de las variables conseguidas a través del modelo de redes neuronales artificiales son coincidentes en el 66,67\% (96/144) de los casos. 
El mayor número de asignaciones se focaliza en las amas de casa; dichas asignaciones representan el $44 \%$ (64/144) del total de casos y el 66,67\% (64/96) del total de asignaciones acertadas.

Tabla 3

Conjunto de evaluación

Matriz de confusión - clases reales (filas) / estimadas (columnas)

\begin{tabular}{|c|c|c|c|c|c|}
\hline \multirow[b]{3}{*}{$\begin{array}{l}\text { Clases } \\
\text { Reales }\end{array}$} & \multicolumn{5}{|c|}{ Variable: OFICIO - ocupación u oficio } \\
\hline & \multicolumn{4}{|c|}{$\begin{array}{c}\text { Asignación según los pesos sinápticos de la red } \\
\text { neuronal }\end{array}$} & \multirow[b]{2}{*}{ Total } \\
\hline & $\begin{array}{c}\text { Ama de } \\
\text { casa }\end{array}$ & $\begin{array}{l}\text { Trabaj. } \\
\text { Indep. }\end{array}$ & Empleada & Pensionada & \\
\hline Ama de casa & 64 & 5 & 0 & 1 & 70 \\
\hline Traba. independiente & 11 & 16 & 0 & 2 & 29 \\
\hline Empleada & 11 & 4 & 7 & 5 & 27 \\
\hline Pensionada & 8 & 1 & 0 & 9 & 18 \\
\hline Total & 94 & 26 & 7 & $17-$ & 144 \\
\hline
\end{tabular}

\subsection{Gustos y preferencias}

Las adultas mayores poseen gustos y preferencias distintos en cuanto al calzado, por cuanto las necesidades propias de este grupo etario derivan de los cambios morfológicos producto de su edad y buscan garantizar que su calzado les brinde comodidad, suavidad, seguridad y buena presentación.

Los resultados de la investigación (tabla 4), muestran que existe coincidencia en la preferencia por zapatos de tela, color negro, con precios asequibles y con una disponibilidad inmediata $(4,4$ a 4,6$)$.

Al analizar estas percepciones en función de la profesión u oficio, se observa que a excepción de los pensionados $(3,8)$ todas las mayores, sin importar su profesión deben realizar una búsqueda extenuante en comercio de la ciudad con el fin de encontrar zapatos que se acomoden a las necesidades de sus pies, a sus preferencias y a su capacidad de pago.

En cuanto a la preferencia por zapatos informales, se observa que las pensionadas y las amas de casa son quienes más tienen afinidad con este tipo de calzado (3,9 y 3,8; respectivamente). Contrastante a lo anterior, las empleadas no muestran una definición clara por este tipo de calzado $(3,2)$; por el contrario, manifiestan tener preferencia por los zapatos cerrados de color café ya que les garantiza la formalidad necesaria y/o exigida en su entorno laboral $(3,6)$.

La aplicación de la prueba F de Snedecor cuyo nivel de significancia se encuentra por debajo del 5\% indica que la percepción que tienen los adultos mayores sobre los precios altos del calzado depende de la profesión u ocio que ocupan.

Por el contrario, la misma prueba (nivel de significancia por encima del 5\%) indica que la percepción de las personas acerca de los zapatos de tela el color negro el uso de zapatos informales, zapatos cerrados formales y la disponibilidad de calzado acorde a los gustos y preferencias, no depende de la profesión u oficio que desempeñan. 
Tabla 4

Gustos y preferencias por el calzado según ocupación u oficio

\begin{tabular}{|c|c|c|c|c|c|c|c|}
\hline \multirow[b]{2}{*}{$\begin{array}{l}\text { № } \\
\text { Var. }\end{array}$} & \multirow[b]{2}{*}{ Denominación } & \multirow[b]{2}{*}{$\begin{array}{c}\text { Total } \\
\text { muestra }\end{array}$} & \multicolumn{4}{|c|}{ Profesión u Oficio } & \multirow[b]{2}{*}{ F de Snedecor } \\
\hline & & & $\begin{array}{l}\text { Ama de } \\
\text { casa }\end{array}$ & $\begin{array}{c}\text { Trabajadora } \\
\text { independiente }\end{array}$ & Empleado & $\begin{array}{c}\text { Pensionad } \\
\text { o }\end{array}$ & \\
\hline \multirow{2}{*}{15} & \multirow{2}{*}{$\begin{array}{l}\text { Zapatos de tela: } \\
\text { comodidad }\end{array}$} & 4,6111 & 4,6714 & 4,6207 & 4,6667 & 4,2778 & $F(3,140)=1,3022$ \\
\hline & & $n=144$ & $n=70$ & $n=29$ & $n=27$ & $n=18$ & $p=0,2762$ \\
\hline \multirow{2}{*}{23} & \multirow{2}{*}{$\begin{array}{l}\text { Zapatos cerrados: } \\
\text { formalidad }\end{array}$} & 3,2778 & 3,0143 & 3,5517 & 3,6296 & 3,3333 & $F(3,140)=1,9599$ \\
\hline & & $n=144$ & $n=70$ & $n=29$ & $n=27$ & $n=18$ & $p=0,1228$ \\
\hline \multirow{2}{*}{24} & \multirow{2}{*}{$\begin{array}{c}\text { Zapatos negros } \\
\text { combinan con todo }\end{array}$} & 4,5347 & 4,4714 & 4,5172 & 4,6296 & 4,6667 & $F(3,140)=0,3210$ \\
\hline & & $n=144$ & $n=70$ & $n=29$ & $n=27$ & $n=18$ & $p=0,8102$ \\
\hline 26 & $\begin{array}{c}\text { Calzado ideal difícil de } \\
\text { conseguir. }\end{array}$ & $n=144$ & $\mathrm{n}=70$ & $n=29$ & $n=27$ & $n=18$ & $p=0,0530$ \\
\hline
\end{tabular}

Fuente: Encuestas - base de datos Bernard K. (2020).

Adulto mayor en Cartagena: necesidades, gustos y preferencias por el calzado

\subsection{Clasificación de gustos y preferencias por el calzado - red neuronal artificial}

Con el fin de obtener una mejor clasificación de los gustos y preferencias del tipo de calzado en relación a la ocupación y oficio de las adultas mayores, se optó por una red neuronal artificial (RNA) utilizando el modelo de clasificación; fueron tomadas como variables output las categorías de la ocupación u oficio y como variables input los gustos y preferencias por el calzado.

El modelo (RNA) perceptrón multicapa, cuenta con tres capas y diez variables en total: seis de entrada y cuatro de salida; por ende, se fija en cinco el número de variables intermedias u ocultas -semisuma del total de variables de input y output-. Figura 2.

Componentes del modelo:

- Numero de interacciones: 100.000

- Tasa de aprendizaje inicial: 1,00

- Tasa de aprendizaje final: 0,70

- Momentos: Inicial: 0,00 - Final: 0,30

- Conjunto de entrenamiento: casos 1 a 144

- Conjunto de Evaluación: casos 1 a 144

La matriz de confusión resultado del análisis (tabla 5), muestra las estimaciones de los valores de las variables obtenidas a través del modelo de redes neuronales artificiales coinciden en el 63,19\% (91/144) de los casos.

Las estimaciones poseen aciertos muy altos en la variable output -ama de casa-, en esta categoría los aciertos en las asignaciones representan el $47 \%$ (68/144) del total de los casos y el 74\% (68/91) del total de asignaciones acertadas. Es menor el porcentaje del total de asignaciones acertadas en el caso de las trabajadoras independientes $12 \%(11 / 91)$ y muy bajo en las pensionadas $7 \%(7 / 91)$ y las empleadas $5 \%(5 / 91)$. 
Figura 3

Redes neuronales artificiales. Gustos y preferencias del adulto mayor por el calzado

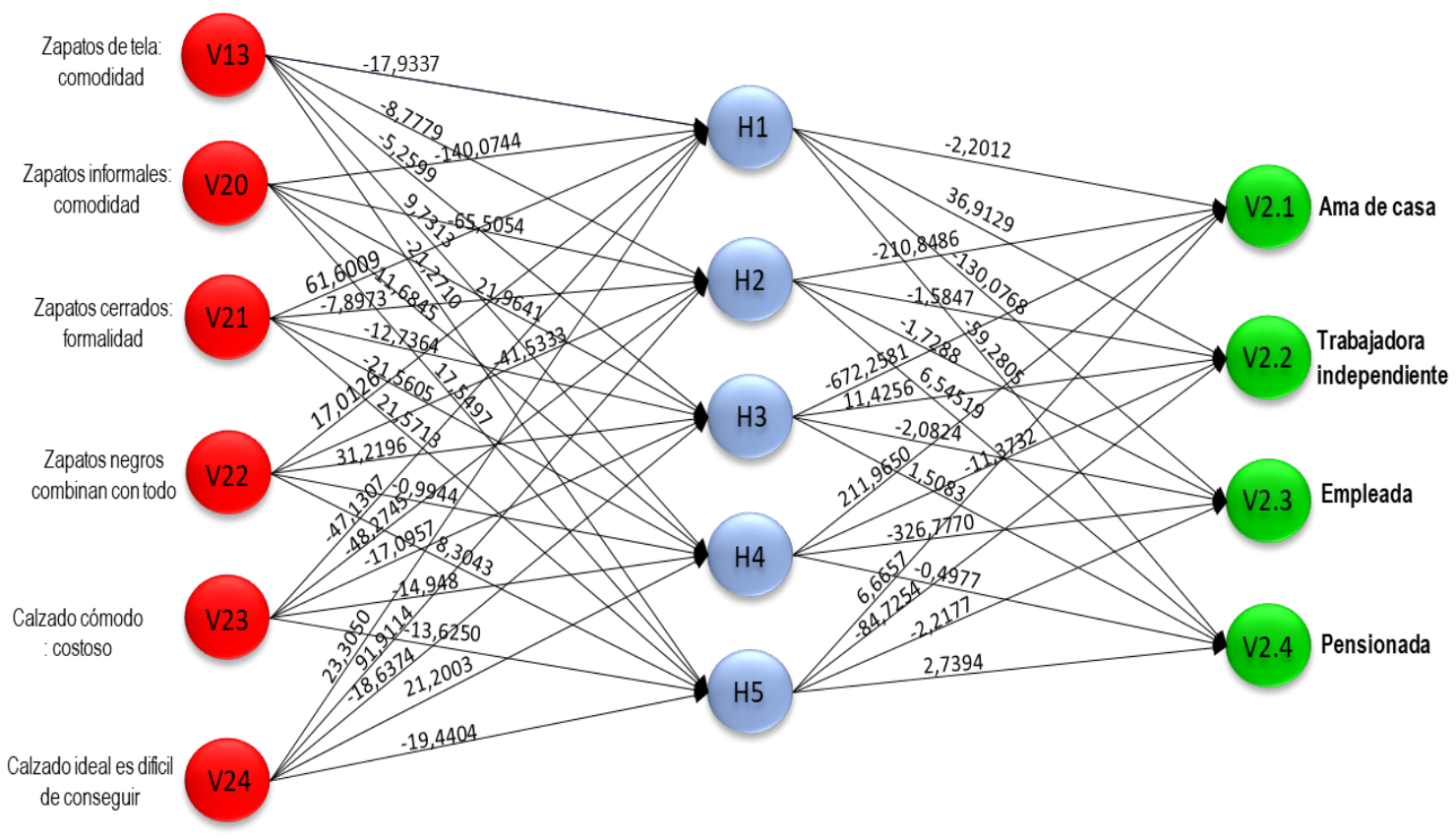

Fuente: Encuestas - base de datos Bernard K. (2020).

Adulto mayor en Cartagena: necesidades, gustos y preferencias por el calzado

Tabla 5

Conjunto de evaluación

Matriz de confusión - clases reales (filas) / estimadas (columnas)

\begin{tabular}{|cccccc}
\hline & \multicolumn{4}{c}{ Variable: OFICIO - profesión u oficio } \\
\hline \multirow{2}{*}{ Clases reales } & \multicolumn{4}{c}{$\begin{array}{c}\text { Asignación según los pesos sinápticos de la red } \\
\text { neuronal }\end{array}$} \\
\cline { 2 - 5 } & $\begin{array}{c}\text { Ama de } \\
\text { casa }\end{array}$ & $\begin{array}{c}\text { Trabajadora } \\
\text { independiente }\end{array}$ & Empleada & Pensionada & Total \\
\hline Ama de casa & 68 & 0 & 1 & 1 & 70 \\
Traba. independiente & 18 & 11 & 0 & 0 & 29 \\
Empleada & 20 & 1 & 5 & 1 & 27 \\
Pensionada & 9 & 1 & 1 & 7 & 18 \\
Total & 115 & 13 & 7 & 9 & 144 \\
\hline
\end{tabular}

Porcentaje de asignaciones acertadas por las redes neuronales: 63,19\%

Fuente: Encuestas - base de datos Bernard K. (2020).

Adulto mayor en Cartagena: necesidades, gustos y preferencias por el calzado

\section{Conclusiones}

Las necesidades de las mujeres adultas mayores en Cartagena de Indias, por el tipo de calzado, al igual que sus congéneres se encuentran principalmente asociadas con el tipo de pie y con los cambios morfológicos que con el paso del tiempo se van presentando; dichos cambios se agudizan en la adultez llegando a convertirse en 
anomalías que contribuyen al deterioro de la calidad de vida; dentro de ellas resaltan los juanetes, callos, uñas encarnadas y dedos de martillo, sin dejar de lado otras afecciones.

La actividad, movilidad, ocupación u oficio de las mayores, determina la necesidad de calzado suave y con forro de almohadilla, para evitar dolencias en los talones y la planta del pie -fascia plantar-.

En el marco de una amplia oferta comercial dirigida a segmentos del mercado de personas jóvenes pero limitada para las de la tercera edad, estas se ven obligadas a buscar de manera intensiva e incluso extenuante calzado acorde con la tipología y los cambios morfológicos de sus pies buscando seguridad y comodidad y en lo posible con diseños que les permitan sentirse bien presentadas. Quienes aún se encuentran laborando prefieren zapatos formales acordes con su entorno profesional y de trabajo en cambio las amas de casa y pensionadas prefieren zapatos de tela y diseño informal.

Los resultados del presente trabajo aportan información para los comerciantes y fabricantes quienes tienen la oportunidad de servir a un segmento con capacidad de pago, que se encuentra en crecimiento y que puede constituirse en un importante nicho de mercado.

\section{Referencias bibliográficas}

Bernard Kathreen. (2020). Adulto mayor en Cartagena: necesidades, gustos y preferencias por el calzado. Trabajo de grado. Universidad de Cartagena.

Carbillet, Pauline (2012): "Étude épidémiologique du type de chaussures de la personne âgée hospitalisée en situation aiguë : relation aux chutes en ambulatoire et à la fragilité au domicile". Semantic.scholar.org.

Chaiwanichsiri, D., Tantisiriwat, N. Janchai, S. (2008): Proper shoe sizes for Thai elderly, Foot Edinb 18 (4) 186191.

DANE. Departamento Nacional de Estadística (2018). Censo Nacional de Población y Vivienda. Colombia.

Droulers, A. y Goldcher, A (2009): “Prise en charge des pathologies du pied d'une personne âgée”, Revista Elsevier, Vol 9, Issue 51, pág. 139-144.

Harvey, Anne (2001): "Les pieds, ces mal-aimés". Le medicine Su Quebec, vol 36, №8.

Jellema, Anton H., Huysmans, Toon., Hartholt, Klaas y Van Der Cammen, Tischa J. (2019). Shoe design for older adults: Evidence from a systematic review on the elements of optimal footwear. Maturitas Vol. 127. Págs 64-81.

Kusumoto, T. Suzuki, H. Yoshida, J. (2008). Kwon, Intervention study to improve quality of life and health problems of community-living elderly women in Japan by shoe fitting and custom-made insoles, Gerontology 53 (6) 348-356.

Jellema, A., Huysmans, T., Hartholt, K., \& van der Cammen, T. (2019). Shoe design for older adults: Evidence from a systematic review for shoe elements required for optimal footwear. Maturitas, 127, 64-81. https://doi.org/10.1016/j.maturitas.2019.06.002

Jellema, A., Huysmans, T., Hartholt, K., \& van der Cammen, T. (2019). Shoe design for older adults: Evidence from a systematic review for shoe elements required for optimal footwear. Maturitas, 127, 64-81. https://doi.org/10.1016/j.maturitas.2019.06.002 
Jellema, A., Huysmans, T., Hartholt, K., \& van der Cammen, T. (2019). Shoe design for older adults: Evidence from a systematic review for shoe elements required for optimal footwear. Maturitas, 127, 6481.https://doi.org/10.1016/j.maturitas.2019.06.002

Losa Iglesias, M.E., de Bengoa Vallejo, Palacios Peña, R., D. (2012). Impact of Soft and hard insole density on postural stability in older adults, Geriatric Nurs. 33 (4) 264-271.

Maki, B.E. (1997). Gait changes in older adults: predictors of falls or indicators of fear? J Am Geriatr Soc. No. 45. p. 313-320.

Marco S., Carmen (2003). Marcha patológica. Revista del pie y tobillo. Vol 17. Núm 1. Sociedad Española de Medicina y Cirugía del Pie y Tobillo

Menz H., Morris M., Lord S.(2006). Footwear characteristics and risk of indoor and outdoor falls in older peopl". Gerontology.;52 (3):174-80. 2006.

Menz, H.B., Auhl, M. S., Frescos Ristevski, N., Munteanu, S.E. (2014): Evaluation of the accuracy of shoe fitting in older people using three-dimensional foot scanning, J. Foot Ankle. Res. 7 (1).

Mulford, D., Taggart, H.M., Nivens, A., Payrie, C., (2008). Arch support use for improving balance and reducing pain in older adults, Appl. Nurs. Res. 21 153-158.

OMS. Organización Mundial de la Salud (2017): Informe mundial sobre el envejecimiento y la salud. A.

Romero S., Manuel, Santalla B., Fátima, Gi M., Pedro, Mosquera F. Abián, Suárez C., María del Carmen y Maceira C., Matilde (2010). Estudio de prevalencia de patologías podológicas y grado de dependencia en mayores de 65 años. El Peu, 30(30). 104-111.

Rueda, Martín (2018): “Los pies: nuestra base y donde empieza todo”. Centre d’Etudis del Peu.

Salech, M. Felipe, Jara, L. Rafael y Michea, A. Luis (2012). Cambios fisiológicos asociados al envejecimiento". Elsevier Journal. Vol 23. Núm. 1.

White, E.G.y Mulley, G.P. (1989): Footwear worn by the over 80's: a community survey, Clin. Rehabil. 3 (1) $23-$ 25. 
Anexos

Redes neuronales para determinar las necesidades de calzado de los adultos mayores según su profesión u oficio.

\begin{tabular}{|c|c|c|}
\hline $\begin{array}{l}\text { Tipo de } \\
\text { variable }\end{array}$ & N.o & Identificación de las variables \\
\hline $\begin{array}{l}\text { Variables } \\
\text { output }\end{array}$ & $\begin{array}{l}1 \text { Ama de casa } \\
2 \text { Trabajador independiente }\end{array}$ & $\begin{array}{l}3 \text { Empleado } \\
4 \text { Pensionado }\end{array}$ \\
\hline $\begin{array}{l}\text { Variables } \\
\text { input }\end{array}$ & $\begin{array}{l}\text { JUANETES - Tengo ju } \\
\text { JUA-SAND - Para los } \\
\text { CALZADO - No se con } \\
\text { CARACPIE - El calzad } \\
\text { FASCIAPL - Tengo la f } \\
\text { Cal-Livi - El calzado li } \\
\text { HORM-CAL - La horm } \\
\text { garra, dedos en mazc } \\
\text { SUE-CAL - Para camir } \\
\text { ENFERPOD - No se co }\end{array}$ & $\begin{array}{l}\text { or lo que necesito calzado suave y holgado. } \\
\text { las sandalias son una buena opción } \\
\text { Izado cerrado cómodo para pies con juanetes. } \\
\text { de acuerdo a las características del pie } \\
\text { antar necesito calzado suave y forro abullonado } \\
\text { ita el cansancio al caminar y permite seguridad } \\
\text { del calzado permite la comodidad de los dedos en martillo, dedos en } \\
\text { uy grueso. } \\
\text { zado debe tener una suela antideslizante. } \\
\text { alzado para pies con dedos llamados: martillo, garra y mazo. }\end{array}$ \\
\hline
\end{tabular}

Número de variables output: 4

Características del modelo

Número de variables input: 9

Número de variables intermedias: 6

Número total de capas: 3

Conjunto de entrenamiento: Registro inicial:1 - Registro final: 144

Conjunto de evaluación: Registro inicial:1 - Registro final: 144

Resultados del entrenamiento de la red Número total de iteraciones $\quad 5.003$

Error cuadrático medio (ECM) mínimo $\quad 0,3420$ Obtenido en la iteración número $\quad 2.380$

Pesos sinápticos (Red con ECM mínimo)

Pesos de los inputs en las funciones de activación de las neuronas intermedias:

\begin{tabular}{|c|c|c|c|c|c|c|}
\hline Variables & $\begin{array}{c}\text { Var. Intermed. } \\
1\end{array}$ & $\begin{array}{c}\text { Var. Intermed } \\
2\end{array}$ & $\begin{array}{c}\text { Var. Intermed } \\
3\end{array}$ & $\begin{array}{c}\text { Var. Intermed } \\
4\end{array}$ & $\begin{array}{c}\text { Var. Intermed } \\
5\end{array}$ & $\begin{array}{c}\text { Var. Intermed } \\
6\end{array}$ \\
\hline Umbral & $-24,5214$ & $-19,6939$ & $-1,5685$ & $-19,1134$ & $-2,3738$ & $-6,0302$ \\
\hline \multicolumn{7}{|l|}{ Variables Input: } \\
\hline JUANETES & 0,8495 & 5,5940 & $-7,4085$ & $-25,6320$ & $-2,5463$ & $-9,8793$ \\
\hline JUA-SAND & 19,8419 & 5,0976 & $-6,1684$ & $-10,1157$ & $-3,2846$ & $-1,3103$ \\
\hline CALZADO & $-10,4406$ & $-9,6066$ & $-3,4539$ & $-7,9602$ & $-13,2494$ & $-4,8822$ \\
\hline CARACPIE & 24,8840 & 0,7935 & 7,3218 & 0,6652 & 3,4389 & $-2,5936$ \\
\hline FASCIAPL & $-37,8083$ & 0,7662 & 1,8899 & 9,8803 & 7,5110 & 22,4158 \\
\hline CAL-LIVI & 12,9295 & $-7,2077$ & $-7,8429$ & $-8,3642$ & $-4,3072$ & 22,3714 \\
\hline HORM-CAL & $-23,8761$ & $-4,5025$ & $-4,3947$ & 7,0984 & 4,8552 & $-29,9622$ \\
\hline SUE-CAL & $-24,6938$ & 6,7471 & $-3,6131$ & 18,0924 & 1,6031 & $-13,5936$ \\
\hline ENFERPOD & $-10,7910$ & $-23,9744$ & 8,0439 & $-33,6183$ & $-12,8765$ & 11,3874 \\
\hline
\end{tabular}

Pesos de las neuronas intermedias en las funciones de activación de los outputs

\begin{tabular}{ccccc}
\hline Variables & OFICIO 1 & OFICIO 2 & OFICIO 3 & OFICIO 4 \\
\hline Umbral & 0,0578 & 16,0608 & 1,6041 & 2,0622 \\
Variables intermedias: & & & & 2,3607 \\
Intermedia 1 & $-8,3055$ & 6,9994 & 0,5067 & $-2,6123$ \\
Intermedia 2 & 22,3833 & $-9,4881$ & $-26,5100$ & 6,8926 \\
Intermedia 3 & 10,4764 & $-13,2229$ & $-13,9789$ & $-5,3795$ \\
Intermedia 4 & $-16,5520$ & 16,5873 & 13,0037 & 7,9721 \\
Intermedia 5 & $-8,9148$ & $-13,6631$ & 18,2281 & $-2,9757$ \\
Intermedia 6 & $-0,5458$ & 15,0408 & $-0,5255$ &
\end{tabular}

Fuente: Encuestas 
Redes neuronales para determinar gustos y preferencias de los adultos mayores por el calzado

\begin{tabular}{ccl} 
Tipo de variable & No & Identificación de las variables \\
\hline \multirow{2}{*}{$\begin{array}{c}\text { Variables } \\
\text { output }\end{array}$} & 1 & Ama de casa \\
& 2 & Trabajador independiente \\
& 3 & Empleado \\
& 4 & Pensionado \\
\hline \multirow{2}{*}{ Variables } & 1 & ZAP-TELA - Los zapatos de tela son más cómodos que los de cuero o sintético. \\
input & 2 & ZAP-INFO - Uso zapatos informales para poder caminar con comodidad \\
& 3 & ZAP-CERR - Uso zapatos cerrados de color café ya que es más formal. \\
& 5 & CAL-NEGR - Prefiero usar calzado negro porque combina con todos los colores de la ropa. \\
& 6 & CAL-COMO - Se consigue calzado muy cómodo, pero es muy costoso. \\
& 6 & CAL-SATI - Para encontrar calzado que me deje satisfecho debo caminar y buscar mucho.
\end{tabular}

Número de variables output: 4

Características del modelo

Número de variables input: 6

Número de variables intermedias: 5

Número total de capas: 3

Conjunto de entrenamiento: Registro inicial:1 - Registro final: 144

Conjunto de evaluación: Registro inicial:1 - Registro final: 144

Resultados del entrenamiento de la red

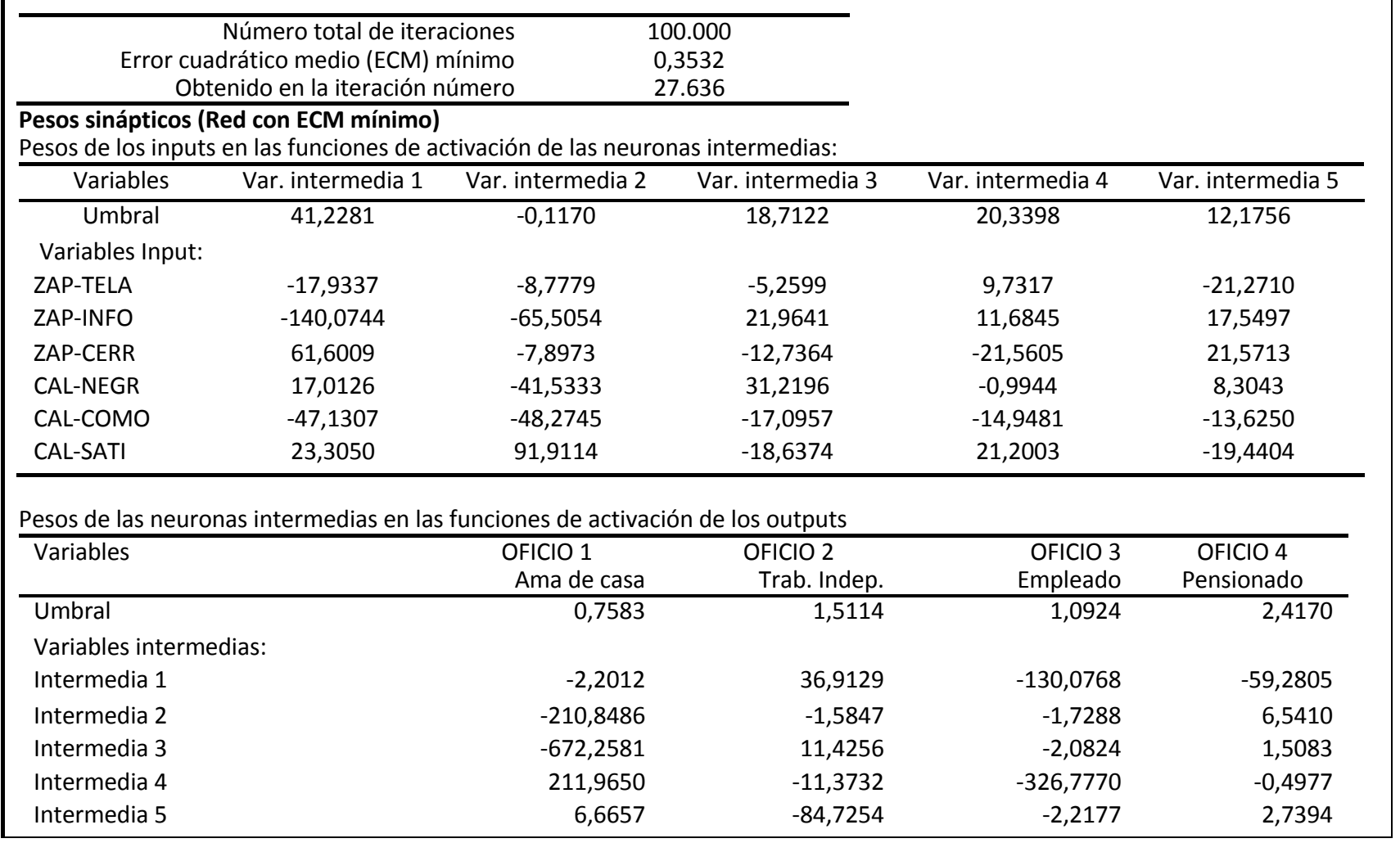

Fuente: Encuestas

Esta obra está bajo una Licencia Creative Commons Attribución-NoCommercial 4.0 International

\section{(cc) BY-NC}

